\title{
Regional wave propagation using the discontinuous Galerkin method
}

\author{
S. Wenk ${ }^{1}$, C. Pelties ${ }^{1}$, H. Igel ${ }^{1}$, and M. Käser ${ }^{2}$ \\ ${ }^{1}$ Department of Earth and Environmental Sciences, Geophysics Section, \\ Ludwig-Maximilians-Universität, Munich, Germany \\ ${ }^{2}$ Geo Risks Research, Munich Re, Munich, Germany
}

Correspondence to: S. Wenk (wenk@geophysik.uni-muenchen.de)

Received: 27 July 2012 - Published in Solid Earth Discuss.: 23 August 2012

Revised: 9 November 2012 - Accepted: 22 November 2012 - Published: 30 January 2013

\begin{abstract}
We present an application of the discontinuous Galerkin (DG) method to regional wave propagation. The method makes use of unstructured tetrahedral meshes, combined with a time integration scheme solving the arbitrary high-order derivative (ADER) Riemann problem. This ADER-DG method is high-order accurate in space and time, beneficial for reliable simulations of high-frequency wavefields over long propagation distances. Due to the ease with which tetrahedral grids can be adapted to complex geometries, undulating topography of the Earth's surface and interior interfaces can be readily implemented in the computational domain. The ADER-DG method is benchmarked for the accurate radiation of elastic waves excited by an explosive and a shear dislocation source. We compare real data measurements with synthetics of the 2009 L'Aquila event (central Italy). We take advantage of the geometrical flexibility of the approach to generate a European model composed of the 3-D EPcrust model, combined with the depthdependent ak135 velocity model in the upper mantle. The results confirm the applicability of the ADER-DG method for regional scale earthquake simulations, which provides an alternative to existing methodologies.
\end{abstract}

\section{Introduction}

To understand the influence of 3-D Earth structure on regional seismic wave propagation (continental scale), numerical algorithms are required that accurately simulate ground motions towards high frequencies using today's computing architectures. The schemes have to flexibly handle compli- cated geological formations, such as the structure of subduction and rifting zones, the shape of mantle plumes, the topography of elastic discontinuities, and the heterogeneities of continental crust. In this study, we present an application of the discontinuous Galerkin (DG) method to regional scale earthquake simulations up to periods of $20 \mathrm{~s}$, offering considerable flexibility to accurately take into account 3-D Earth models particularly in the meshing part of the workflow.

The first regional to global scale simulations based on spherically symmetric Earth models, were obtained by the summation of normal modes (Alterman et al., 1959). The approach was extended to account for effects of 3-D Earth structures, and localized heterogeneities using perturbation theory (e.g., Woodhouse, 1983). Besides semi-analytical algorithms, full numerical schemes evolved, such as the finitedifference (FD) method. The FD method approximates solutions to the wave equation on a structured grid, applying low-order (e.g., Virieux, 1984), and later also high-order (e.g., Bayliss et al., 1986) numerical operators in 3-D, in order to improve the accuracy and computational efficiency. Due to restrictions in the geometrical flexibility of standard FD methods, applying structured grids with regular grid spacing, approaches which make use of variable grids have also been introduced (Pitarka, 1999; Ely et al., 2008; Kristek et al., 2010; Kozdon et al., 2012).

As an alternative, finite-element (FE) methods have been applied in seismology (e.g., Bao et al., 1998). The techniques make use of orthogonal basis functions to approximate the solution in finite subspaces (elements) of the modeling domain. This concept enables the use of various element types (e.g., tetrahedra, hexahedra, pyramids, prisms 
and combinations of them) to flexibly discretize the computational domain.

A particular FE scheme is the spectral-element (SE) method, which is state-of-the-art in regional and global earthquake simulations at the moment (e.g., Priolo et al., 1994; Komatitsch, 1997; Seriani, 1998; Chaljub, 2000; Capdeville et al., 2003; Nissen-Meyer et al., 2008; Fichtner et al., 2009; Cupillard et al., 2012). The SE method supports computational grids of deformable hexahedral elements, to simulate fully 3-D wavefields with high-order spatial accuracy, and explicit time extrapolation schemes.

Motivated by the difficulties in mesh generation for complex geometries using structured or unstructured deformable hexahedral grids, the discontinuous Galerkin method was introduced to extend the modeling capabilities to unstructured tetrahedral grids (e.g., Käser and Dumbser, 2006; Dumbser and Käser, 2006; Käser et al., 2007; de la Puente et al., 2007; Dumbser et al., 2007, and Etienne et al., 2010). To honor complex 3-D shapes, tetrahedra offer the largest flexibility, because the elements can easily be aligned to small scale structures of the Earth, and the size of the elements can be locally adapted ( $h$-adaptivity). Furthermore, the adaptation of the polynomial degree inside each element ( $p$-adaptivity) enables the computational load to be focused on areas of interest. In addition to the spatial flexibility, the accumulation of errors, due to numerical dispersion, needs to be minimized. Therefore, an arbitrarily high-order derivative (ADER) scheme (Toro, 1999) was combined with the DG method for elastodynamic problems to achieve the same accuracy for the temporal, as for the spatial approximation of the wavefield. The ADER-DG approach supports local timestepping schemes to balance the varying computational costs of elements with different size and polynomial order (Dumbser et al., 2007).

In this paper, we show that the ADER-DG method can serve as an alternative algorithm to established numerical methods for regional-scale earthquake simulations. In particular, we investigate the applicability of the approach in terms of mesh generation and simulation accuracy.

The paper is structured as follows. In Sect. 2 we describe the proposed ADER-DG approach for the numerical simulation of wave propagation in 3-D media. In addition to the numerical properties of the ADER-DG implementation, we explain in detail the mesh generation process for geometrically complex 3-D media. In Sect. 3 we verify the radiation of elastic waves excited by an explosive and a shear dislocation source. Synthetics of the SE and our ADER-DG approach calculated in the 1-D isotropic PREM model (Dziewonski and Anderson, 1981) are compared. Finally, in Sect. 4 we show numerical results obtained in a composite model of Europe, where the 3-D crustal model EPcrust (Molinari and Morelli, 2011) is imposed on top of the 1-D reference Earth model ak135 (Kennett et al., 1995), and compare them to observations.

\section{Wave propagation in 3-D media}

\subsection{The ADER-DG approach}

To give an introduction to the numerical scheme of the ADER-DG method, we will qualitatively describe the features and usability of the approach with a special focus on the model discretization. For details on the theoretical framework and the implementation of the method the reader is referred to the work of Käser and Dumbser (2006); Dumbser and Käser (2006); Käser et al. (2007); de la Puente et al. (2007), and Dumbser et al. (2007).

Like every finite-element method, the DG method solves the weak form of a partial differential equation, here the elastic wave equation. Therefore, the wave equation is multiplied by a test function, and integrated over a finite element subspace. In our implementation of the DG scheme, the complete 3-D computational domain can be divided into a mesh of unstructured tetrahedra. The unknown solution is approximated inside each element by a linear combination of spacedependent polynomial basis functions of arbitrary degree $N$, and its purely time-dependent coefficients, the so-called degrees of freedom (DOF), which advance in time. DG is referred to as a Galerkin scheme, as most of the FE-type methods used in computational seismology (e.g., the classical FE and SE methods), because test functions are chosen from the same space-dependent basis function. We use an orthogonal modal basis suggested by Dubiner (1991).

In contrast to classical FE and SE schemes, the polynomial representation of the physical solution is not forced to be continuous across element boundaries in DG. At this point, the DG approach adopts the well-known concept of fluxes to exchange information between neighboring elements, as defined in the finite-volume (FV) approach. The flux terms are computed via the upwinding exact Riemann solver, known as the Godunov flux (Toro, 1999). Note that differently from high-order FV schemes only adjacent elements communicate, and a reconstruction process is not required. Furthermore, the amount of data, necessary for the flux computation, reduces to a rather small number of DOF. In case of a parallel run only minor MPI communication occurs, and the code is highly scalable (Käser et al., 2010).

The method can be implemented efficiently, since, in the discrete form of the scheme, the occurring mass, stiffness, and flux matrices can be precomputed. Therefore, each individual shaped and located tetrahedron is transformed from the physical space into a uniform reference element.

The elastic material properties vary in the subsurface depending on the physical model applied. In our approach, elastic parameters are averaged over each vertex of an element, and only a single value is assigned to the entire element. This piece-wise constant approximation simplifies the implementation, but it is not an intrinsic limitation of the scheme, as shown by Castro et al. (2010). 
The quadrature free DG discretization is advanced in time by the arbitrary high-order derivatives (ADER) approach of Toro (1999). A Cauchy-Kovalewski procedure is applied to recursively replace the temporal derivatives in the Taylor expansion by spatial derivatives. In this way, we obtain automatically the same order of accuracy for the time integration as well as for the spatial approximation, while allocating the same memory as a first-order explicit Euler time integration. The code has been proven to achieve spectral convergence (Dumbser and Käser, 2006).

\subsection{Local adaptivity and load balancing}

In our implementation of the ADER-DG method, unstructured, tetrahedral grids can easily be refined to local structures ( $h$-adaptivity). This increases the spatial sampling of geometrical complex structures or the wavefield itself. Within the computational domain, the size of the elements can vary tremendously without any known restrictions (e.g., up to an element size ratio of 1000 in Dumbser et al., 2007), depending on the problem and the refining strategy. Corollary, the time increment according to the CourantFriedrichs-Lewy (CFL) condition can change dramatically between the elements. In order to reduce the computational effort, and balance the different time-step lengths, the polynomial degree can be adapted in each tetrahedral element individually ( $p$-adaptivity). Furthermore, computational efficiency can be enhanced using an optimal time-step length for each individual element (local time stepping). This enables the use of elements with completely different sizes, without affecting the high-order accuracy of the method (Dumbser et al., 2007).

On the other hand, asynchronous element updates due to local time stepping combined with a $p$-adaptive mesh can cause irregular load balancing on parallel machines. This issue can be handled by zoning equally-sized elements of similar material properties, and dividing these individual domains using the graph-partitioning technique of METIS (Karypis and Kumar, 1998), according to the total number of computational tasks. This aims at an equal distribution of small and large elements over all processing units, to balance the workload (Käser et al., 2010).

Since the zones are assigned randomly to each task, exchanging updated field values can increase MPI communication. However, compared to the numerous calculations inside each domain, and the small MPI communication effort for DG, this overhead is small (Dumbser and Käser, 2006).

\subsection{Mesh generation in geometrically complex 3-D media}

Geoscientific models include geometrically complex 3-D structures, such as rough surface topography, as well as complicated undulating interfaces at subsurface velocity contrasts that governs the the propagation of seismic waves.
The generation of a computational mesh incorporating such features can be the most time-consuming part of the whole forward modeling process. Therefore, we want to review different meshing concepts, with respect to the model complexity, using structured and unstructured meshing schemes. Structured meshing denotes the subdivision of the computational domain in a regular-spaced mesh. Unlike structured meshes, unstructured meshes are composed by an irregular pattern of elements, which are not arranged in a logical sequence.

Furthermore, we want to define the explicit and implicit meshing paradigms, which can be applied in structured as well as unstructured meshing schemes. Explicit meshing denotes the concept of adjusting element boundaries exactly along known material interfaces of the physical model. In contrast, implicit meshing schemes do not respect internal material discontinuities in the mesh.

The meshing approach, with respect to the approximation of the material values, can significantly influence runtime and simulation accuracy in consideration of the numerical method. Recent studies investigated whether internal material discontinuities of complex 3-D structural models should be geometrically respected by the mesh or not (Komatitsch et al., 2004; Lee et al., 2008; Casarotti et al., 2008a; Stupazzini et al., 2009; Pelties et al., 2010; Cupillard et al., 2012). In general, the explicit meshing of complex boundaries leads to the most accurate results. However, using an implicit representation, comparable results can be obtained at a lower meshing effort, but at much higher computational costs.

\subsubsection{Structured meshing}

Structured meshing is an approach of discretizing a geological subsurface model, which is computationally very efficient to implement. The structured sampling of material properties at FD gridpoints, FE integration nodes or complete elements usually allows only for an implicit discretization strategy at curved boundaries, which results in a staircase approximation of the Earth's surface or complex internal interfaces. This leads to first-order errors proportional to the grid spacing or time step, that are insensitive to the approximation order of the numerical scheme (Gustafsson and Wahlund, 2004). To achieve high accuracy of the simulation, the regular grid spacing is forced to be very small, leading to an increasing computational effort especially for strong material heterogeneities (Bohlen and Saenger, 2006; Pelties et al., 2010).

There are more sophisticated approaches to implicitly incorporate material interfaces using structured grids. For example, Kristek and Moczo (2003) are using values of effective grid material parameters to account for discontinuities.

For regional wave propagation problems in 3-D, numerical algorithms evolved solving the wave equation efficiently in spherical coordinates of smaller sections of the Earth, using 
curved hexahedral grids (Igel, 1999; Igel et al., 2002; Fichtner and Igel, 2008; Fichtner, 2009). The spherical discretization leads to singularities in the solution of the wave equation, which inhibits an application to fully global simulations. Therefore, the cubed sphere approach (Ronchi, 1996) was introduced, which became very successful, in particular in combination with the SE method (Komatitsch and Tromp, 2002).

Hereby, the projection scheme is based on mapping a cube, divided by an equidistant quadrilateral grid, to an angulardistant quadrilateral grid at the surface of a sphere. In radial direction, interpolation produces hexahedra with constant size while the lateral size decreases towards the center of the sphere, due to the spherical geometry. This results in an unnecessary oversampling of the wavefield, since, generally, velocity values increase with depth. To avoid this problem, the mesh is re-gridded to double the mesh spacing at different radial depths. Furthermore, grid doubling techniques have the potential to improve efficiency, as the total number of elements can be reduced. However, grid doubling is difficult to implement, and can produce undesired numerical artifacts, like instabilities or spurious reflections at the grid interfaces.

The cubed sphere approach and spherical discretizations overcome the limitations of implicit meshing of spherical interfaces, and can respect strong material discontinuities explicitly by element boundaries in a structured mesh. However, the simple implicit, structured meshing approach, we described at the beginning, is still widely used.

\subsubsection{Unstructured meshing}

In contrast to structured meshes, the elements in an unstructured mesh have to be defined by a list of vertex coordinates and its connectivity to the elements, which increases the algorithmic complexity of corresponding numerical approaches. Furthermore, deriving a scheme that is as accurate and efficient as a method implemented on structured grids is challenging. But on the other hand, a robust flexibility in mesh generation of complicated 3-D structures can be gained. Therefore, to utilize the flexibility of the approach and to increase numerical accuracy, unstructured meshing is mainly performed explicitly.

In general, unstructured grids for complex structures cannot be obtained automatically, and a meshing tool is required. An external meshing software such as Cubit (Blacker et al., 1994) can be employed to generate unstructured hexahedral grids, for example, for regional seismological applications (see Casarotti et al., 2008b; Stupazzini et al., 2009; Peter et al., 2011; Cupillard et al., 2012).

The generation of high-quality unstructured hexahedral meshes, as applied in the examples given above, often requires a strong user interaction, which is time consuming and cumbersome. FE and FV methods, for example, can make use of fully unstructured grids with various element types, but can usually be implemented efficiently only in low-order formulations that are very dispersive. This motivated the development of numerical methods that can handle unstructured tetrahedral meshing approaches, such as the high-order accurate DG method.

Especially for arbitrary shaped 3-D models including, for example, interface and surface topography, tetrahedral grids are much more flexible to adapt. This tremendously reduces the meshing effort, but potentially at the expense of longer simulation time due to the overhead generated by the bookkeeping of the element nodes and their connectivity. On the other hand, the extensive use of mesh coarsening capabilities using DG on unstructured tetrahedral meshes, as is shown for an exploration scale study in Käser et al. (2010), can save numerical costs. Whether a net reduction of computational time is achieved or not is highly dependent on the given problem setup.

\subsection{Workflow of tetrahedral mesh generation using Cubit}

The general workflow to generate computational meshes can be divided into the geometry setup and the meshing process itself. For both parts we use the commercial software $\mathrm{Cu}$ bit. This meshing tool contains numerical libraries to represent the underlying geometry of a computational domain using non-uniform rational B-splines (NURBS) that can accurately describe arbitrary geometrical shapes. Furthermore, it provides a flexible tetrahedral meshing algorithm to generate high-quality meshes.

In the following subsections, the geometry and mesh generation workflow using Cubit is demonstrated for an Earth model of Europe (Sect. 4), composed of the 3-D EPcrust model (Molinari and Morelli, 2011) imposed on the depth dependent $a k 135$ velocity model (Kennett et al., 1995).

\subsubsection{Geometry generation - European model}

Implicit meshing of strong material contrasts requires a high resolution for our ADER-DG implementation, because a constant material approximation is used. Consequently, the computational costs could be unnecessary expensive, although the dispersion properties would allow for larger elements. In order to reduce the runtime, improve the accuracy of model approximation, and utilize the flexibility of tetrahedral meshing, we respect interfaces such as the Moho or upper-mantle discontinuities in the European model.

For 3-D models, these interfaces often are given by structured or unstructured point clouds, which have to be composed by parametric surfaces. For example, the 3-D EPcrust model is parametrized by angular-distant points given on a structured grid of $\left(0.5^{\circ} \times 0.5^{\circ}\right)$ and variable depth. It is provided in a TomoJSON format, proposed by the NERIES project (Network of Research Infrastructures for European Seismology). The file contains the material information and 


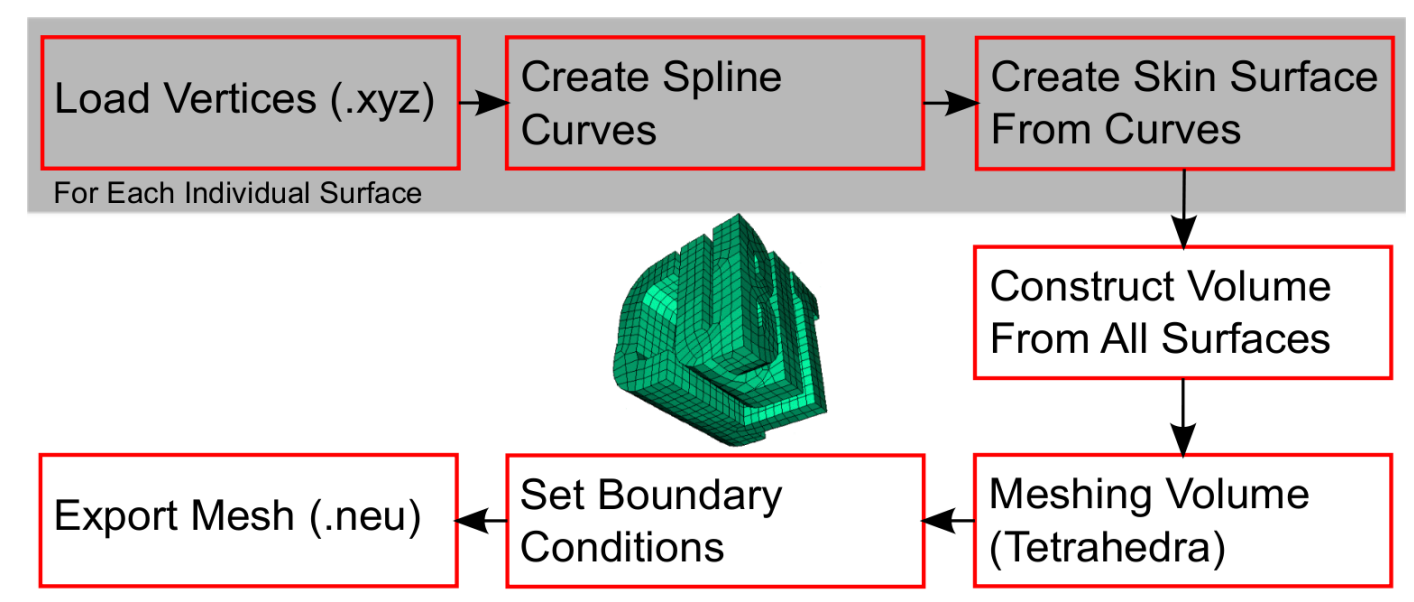

Fig. 1. Workflow of the mesh generation process using Cubit.

locations of the 3-D interfaces. The upper-mantle discontinuities of the ak135 model are represented in the model as smooth spherical shells. The surfaces are reconstructed in Cubit using the radius of the respective discontinuity and the same lateral sampling points of the crustal interfaces.

The geometrical representation of the European model is shown on the left side of Fig. 5. The pointset describing the topography and each individual interface in the model can be imported into Cubit from an ASCII file, including a list of vertex locations in Cartesian coordinates. To reconstruct the surface a set of parallel spline curves along a row or column of a pointset has to be created using the vertices as a spatial support. From the generated lineset, an accurate representation of the surfaces can be reconstructed within Cubit. For large datasets these steps can be automated using the Pythoninterface of Cubit.

NURBS surfaces do not necessarily coincide with their local control points. However, in the case of the reconstruction of the 3-D Moho discontinuity, the distance in normal direction of the parametric surface to points given by the EPcrust model is less than $0.01 \%$ relative to their absolute location. Furthermore, the spacing of the pointsets is smaller than the element size, whereby mesh spacing determines the accuracy of the surface reconstruction.

For each layer of the model, corners of upper and lower surfaces are connected by line segments to generate the boundary faces of the domain. Therefore, interfaces and boundaries are connected at common edges and a closed volume can be obtained.

As part of the workflow, the geometry generation is summarized by the first four boxes in the flowchart depicted in Fig. 1. The frames highlighted in gray illustrate the surface reconstruction, which was performed for each model interface separately.

As already mentioned, the pointsets representing subsurface structures of the Earth can be arbitrarily distributed.
Therefore, it is necessary to use robust surface reconstruction algorithms. Computer-aided design (CAD) tools focused on geometry generation may be an alternative to generate surfaces directly from pointsets, but in most cases these are commercial packages.

\subsubsection{Tetrahedral mesh generation - European model}

Mesh generation and in particular the size of elements inside different areas of the computational domain depend on three main factors: (1) the wavefield approximation, (2) the approximation of the material model, and (3) the approximation of the geometrical model.

In Cubit the tetrahedral mesh generator GHS3D (George et al., 1990) is used to fill arbitrary 3-D volumes with tetrahedral elements. But surfaces first have to be triangulated, for example, by a Delaunay triangulation.

Cubit also provides sizing functions to control the element size and its growth rate in a volume during meshing, where the element size is defined by its edge length. This allows for a local mesh refinement or coarsening, considering a wellbalanced aspect ratio ( $\mathrm{AR}=r_{c} / 3 r_{i}$, with $r_{c}$ circumsphere radius and $r_{i}$ insphere radius) of the tetrahedral elements. In addition, quality measures can provide information on the shape of the elements. Statistics on the mesh elements are necessary because element distortions can lead to very small time steps, increasing the computational costs or even leading to instabilities interrupting the numerical simulation.

The right side of Fig. 5 shows the result of the mesh generation for the European model. Meshing tests of different constant element sizes have shown a strong dependency of the mesh quality on the chosen edge length of the elements, even if little changes to the element size were applied. This was particularly apparent for crustal meshes, since the ratio of the element edge length and the layer thickness is small. In cases the aspect ratio of elements has been large $\left(r_{c} \gg 3 \cdot r_{i}\right)$, 
Table 1. Mesh statistics European mesh.

\begin{tabular}{lccc}
\hline $\begin{array}{l}\text { Subvolume } \\
\text { (start/end depth) }\end{array}$ & $\begin{array}{c}\text { Number of } \\
\text { elements }\end{array}$ & $\begin{array}{c}\text { Edge length } \\
\text { min/max }\end{array}$ & $\begin{array}{c}\text { Aspect ratio } \\
\text { min/max }\end{array}$ \\
\hline crust/Moho & 484768 & $13 / 18 \mathrm{~km}$ & $1.0 / 5.0$ \\
Moho/80 km & 463232 & $20 / 25 \mathrm{~km}$ & $1.0 / 3.7$ \\
$80 \mathrm{~km} / 210 \mathrm{~km}$ & 714213 & $20 / 25 \mathrm{~km}$ & $1.0 / 3.4$ \\
$210 \mathrm{~km} / 410 \mathrm{~km}$ & 1067948 & $22 / 26.5 \mathrm{~km}$ & $1.0 / 3.4$ \\
$410 \mathrm{~km} / 660 \mathrm{~km}$ & 965513 & $28 / 31.5 \mathrm{~km}$ & $1.0 / 3.7$ \\
\hline Total & 3695674 & $13 / 31.5 \mathrm{~km}$ & $1.0 / 5.0$ \\
\hline
\end{tabular}

re-meshing with a different element size or manual changes made to the mesh nodeset have improved mesh quality.

For a numerical simulation, boundary conditions have to be assigned to the corresponding element faces. In our implementation of the DG method, absorbing boundary conditions are applied (Käser and Dumbser, 2006). Together with a list of the connectivity between vertices and elements, a list of boundary elements is produced and converted into a mesh file used by our ADER-DG solver SeisSol. The mesh generation is summarized by the last three boxes in the flowchart, Fig. 1.

We emphasize that despite the mentioned difficulties, the generation of a high-quality tetrahedral mesh took one day for the given example (see statistics of mesh generation in Table 1), which clearly indicates the flexibility of unstructured tetrahedral meshing for complex 3-D models. Furthermore, a strategy to provide a mesh generation workflow using noncommercial software has further to be elaborated.

\section{Benchmarks}

To verify the accuracy of our ADER-DG solver SeisSol on a regional scale, synthetic seismograms are compared to solutions of the well-established SE method implementation SpecFEM3D-Globe. For regional to global scale simulations, SpecFEM was successfully benchmarked against quasianalytical normal mode solutions (Komatitsch and Tromp, 2002).

In the first experiment, we investigate elastic wave propagation excited by an explosive source. Afterwards, the radiation of seismic waves is studied for a shear dislocation source.

In SeisSol as well as in SpecFEM, the source can be located at an arbitrary position inside the element, and does not necessarily have to coincide with a mesh point or Gaussian integration node. But, since different polynomial basis functions are used, the discrete representation of the source term is different. In SeisSol, the basis functions are evaluated directly at the source position, whereas in SpecFEM the point source gets spread over the entire source element. If the source point coincides with an element boundary or ver- tex, only one element is allowed to contain the source. For the simulations, in both schemes a spatial approximation order of $\varnothing=5$ is chosen. Whereas the ADER approach provides the same order of spatial and temporal accuracy, the SE method uses a time integration scheme of second order accuracy. Ground displacements calculated by SpecFEM are differentiated in time to compare them to the velocity output of SeisSol. With respect to the results of the SE method, a seismogram difference can be quantified by the time-frequency misfit analysis introduced by Kristekova et al. (2006). Here, the signals are decomposed in the time-frequency plane to characterize the difference by separation of phase and envelope misfits.

\subsection{Explosive source}

\subsubsection{Setup}

In this test, we analyze the numerical radiation of seismic waves excited by an explosive source using SeisSol. The point dislocation is represented by a moment tensor where the only non-zero entries are $M_{x x}=M_{y y}=M_{z z}=10^{18} \mathrm{Nm}$. The origin of the coordinate system is located in the center of the Earth at $\left(x_{0}, y_{0}, z_{0}\right)=(0 \mathrm{~km}, 0 \mathrm{~km}, 0 \mathrm{~km})$. The source is located at $\left(x_{s}, y_{s}, z_{s}\right)=(0 \mathrm{~km}, 0 \mathrm{~km}, 6361 \mathrm{~km})$, which corresponds to a depth of $10 \mathrm{~km}$ beneath the North Pole. The source time function (STF) is given by a Ricker wavelet with a dominant period of $T=20 \mathrm{~s}$, which we want to accurately recover in our simulations. Note that the signal contains a considerable amount of energy within the range of approximately half the dominant period. Since these low periods can be distorted by the simulation, the synthetics are low-pass filtered.

The physical domain of the SeisSol simulation is a cuboid of $\Omega=[-1000 \mathrm{~km}, \quad 1000 \mathrm{~km}] \times[-500 \mathrm{~km}, \quad 3500 \mathrm{~km}] \times$ $[2400 \mathrm{~km}, 6400 \mathrm{~km}]$ cut by spherical interfaces at depths according to the elastodynamic discontinuities of PREM (see Fig. 3.3). In fact, we consider the complete 1-D isotropic PREM model with an uppermost fluid layer replaced by crustal material. The spherical sections $\Omega_{i}$ are meshed separately. Here, the flexibility of the unstructured mesh is exploited to map material gradients within $\Omega_{i}$ to corresponding changes in the element size. This keeps a constant number of 3 tetrahedral elements per shortest wavelength in each subdomain to model a dominant wave period of $T=20 \mathrm{~s}$. We applied a severe spatial discretization of the mesh to ensure a very high accuracy of the simulation.

SpecFEM provides a mesh for the whole Earth adapted to the PREM model. The number of spectral elements at the Earth's surface, along one side of a cubed sphere chunk (1/4 of Earths perimeter), is set to 384 . Due to the spherical geometry, the mesh is doubled in size three times towards the Earth's core. According to benchmark results of Komatitsch and Tromp (2002), the setup allows for the simulation of $T_{\min }=12 \mathrm{~s}$ as a dominant wave period. 


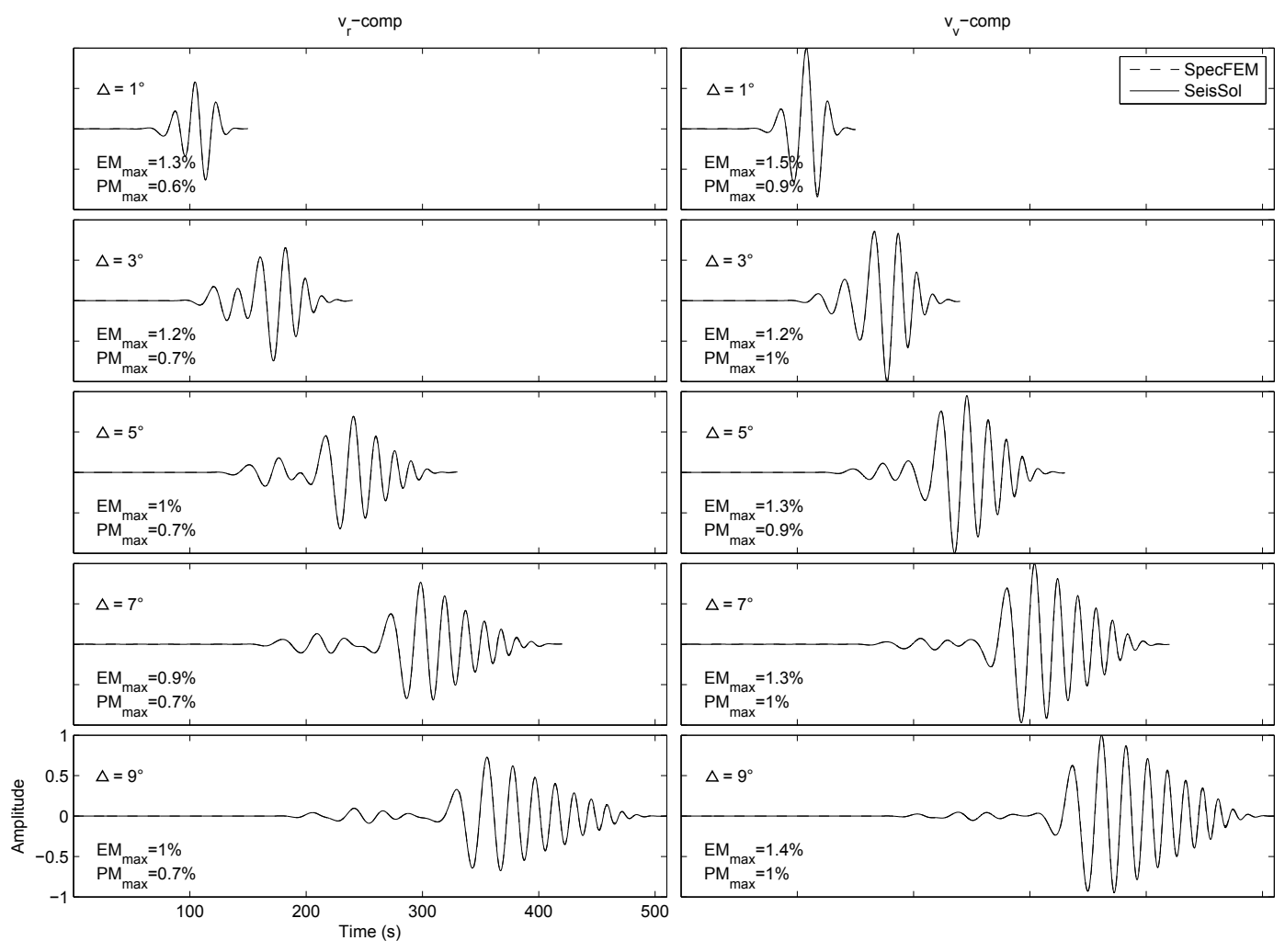

Fig. 2. Synthetic seismograms obtained on the 1-D isotropic PREM model by the ADER-DG (solid) and spectral-element method (dashed) compared at epicentral distances $\Delta$ for an explosive source located at the North Pole. The data are convolved with a Ricker source time function and filtered to periods of $T \geq 20 \mathrm{~s}$. Amplitudes of the radial $v_{r}$ and vertical $v_{v}$ velocity component are normalized with respect to $v_{v}$. The fit between the different synthetic results is quantified by the phase (PM) and envelope (EM) time-frequency misfits. Artificial boundary reflections are cropped.

At five stations, which are located at latitudes from $89^{\circ} \mathrm{N}$ to $81^{\circ} \mathrm{N}$ and longitude $0^{\circ} \mathrm{W}$, seismograms of $800 \mathrm{~s}$ are produced as output. The propagation distance in terms of the number of wavelengths between source and receivers gives $\# \lambda=16$. Data of SeisSol and SpecFEM are filtered for long period signals at a cut-off period of $20 \mathrm{~s}$ using an order three Butterworth filter.

\subsubsection{Results}

Figure 2 shows synthetics of the radial $\left(v_{r}\right)$ and vertical velocity component $\left(v_{v}\right)$ as a function of time. For each station, the traces are normalized with respect to the $v_{v}$ component, which is excited most strongly by the explosive source.

The signals of body and surface waves simulated by SeisSol and SpecFEM match almost perfectly at all stations. This visual impression is confirmed by low values of phase and envelope misfits. Envelope misfits vary around $0.9-1.5 \%$, while phase misfits do not exceed the level of $1 \%$. The lower phase misfits indicate a lower sensitivity of SeisSol to numerical dispersion effects but stronger numerical dissipation, which was already determined by Käser et al. (2008) who compared the algorithm to analytical solutions. Note that the synthetics of SeisSol and SpecFEM are both prone to phase and amplitude misfits within their numerical accuracy.

In case of an explosive source excitation, transverse motion (not shown) should be recorded only at the numerical noise level, which holds for the SE method simulation. However, in the $v_{t}$ component of the SeisSol output clear waveform signals emerge, which gain up to $5 \%$ of the peak amplitude at the $v_{v}$ component. This is suspected to be caused by the application of unstructured grids and will be discussed more in detail in Sect. 3.3.

\subsection{Shear dislocation source}

\subsubsection{Setup}

The main purpose of this simulation is to verify accurate numerical wave radiation in SeisSol using a shear dislocation source, again applied to the 1-D isotropic PREM model. We chose a magnitude 6.0 event, which occurred on the 20th of August 2009 (06:35 GMT) in the Norwegian Sea at latitude $72.22^{\circ} \mathrm{N}$ and longitude $0.84^{\circ} \mathrm{E}$ at a depth of $12 \mathrm{~km}$. The six independent components of the centroid moment tensor (CMT) solution are given in a spherical reference system 


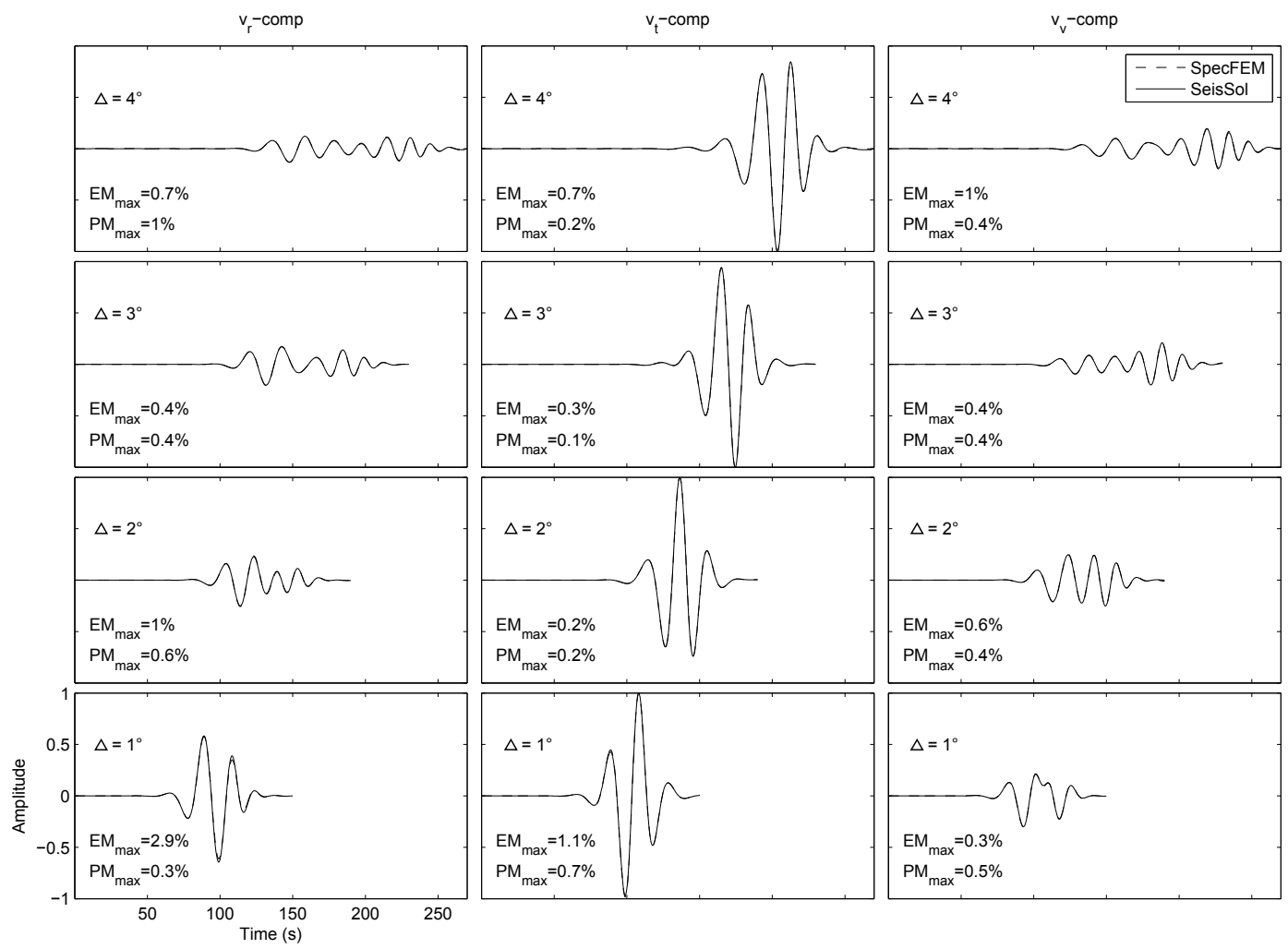

Fig. 3. Synthetic seismograms obtained on the 1-D isotropic PREM model by the ADER-DG (solid) and the spectral-element method (dashed) compared at epicentral distances $\Delta$ for a real source located in the Norwegian Sea. The data are convolved with a Ricker source time function and filtered to periods of $T \geq 20 \mathrm{~s}$. Amplitudes of the radial $v_{r}$, transverse $v_{t}$ and vertical $v_{v}$ velocity component are normalized with respect to $v_{t}$. The fit between the different synthetic results is quantified by the phase (PM) and envelope (EM) time-frequency misfits. Artificial boundary reflections are cropped.

provided by the CMT catalog of the Lamont-Doherty Earth Observatory, Columbia University, see Table 2.

For the SeisSol simulation, we adapted the size of the block model of Sect. 3.1 around the source region to a spherical domain of $\Omega_{\text {surf }}=\left[67^{\circ}, 77^{\circ}\right] \times\left[-5^{\circ}, 5^{\circ},\right]$ with a depth range of $500 \mathrm{~km}$. Except for the source characteristics, SpecFEM results correspond to the same setup as in the previous example. The propagation distance in terms of the number of wavelengths between source and receivers gives $\# \lambda=8$. At four stations located at latitudes from $78^{\circ} \mathrm{N}$ to $73^{\circ} \mathrm{N}$ and longitude $0^{\circ} \mathrm{W}$, synthetic seismograms of $350 \mathrm{~s}$ length are recorded. The signals are processed using the same STF and low-pass filter as specified in Sect. 3.1.

\subsubsection{Results}

Figure 3 illustrates the velocity components plotted as a function of time at stations northward of the source. Here, amplitude values are normalized to the strongest transverse velocity component $v_{t}$. As expected, the signals of SeisSol and SpecFEM are almost identical. Except for near offset stations, the envelope and phase misfits have largest values of $1 \%$, where in general, as for the first experiment, the enve- lope misfit level is higher than the phase misfit. The large envelope misfit value of $2.9 \%$ at station $X 73 \mathrm{~N} .0$, which is closest to the source, could be due to a different excitation characteristic of tetrahedral compared to hexahedral element.

\subsection{Discussion}

Accurate simulations of elastic wave propagation using the ADER-DG approach could be verified applying different source mechanisms on regional sections of the 1-D isotropic PREM model. The overall match of waveforms simulated by SeisSol compared with SpecFEM results are excellent.

However, at far offset stations a remaining, very low envelope and phase misfit of around $1 \%$ is observed. The misfit values represent the expected accuracy of the simulations. However, the different sampling of the material values of SeisSol and SpecFEM has an influence on the numerical accuracy, and could explain small differences in the results. As already mentioned, in SeisSol a constant value for each material parameter is averaged over all vertices in one single tetrahedral element, whereas in SpecFEM material values are evaluated at each GLL point within a hexahedron, leading to a higher (subcell) sampling of the PREM background model. 


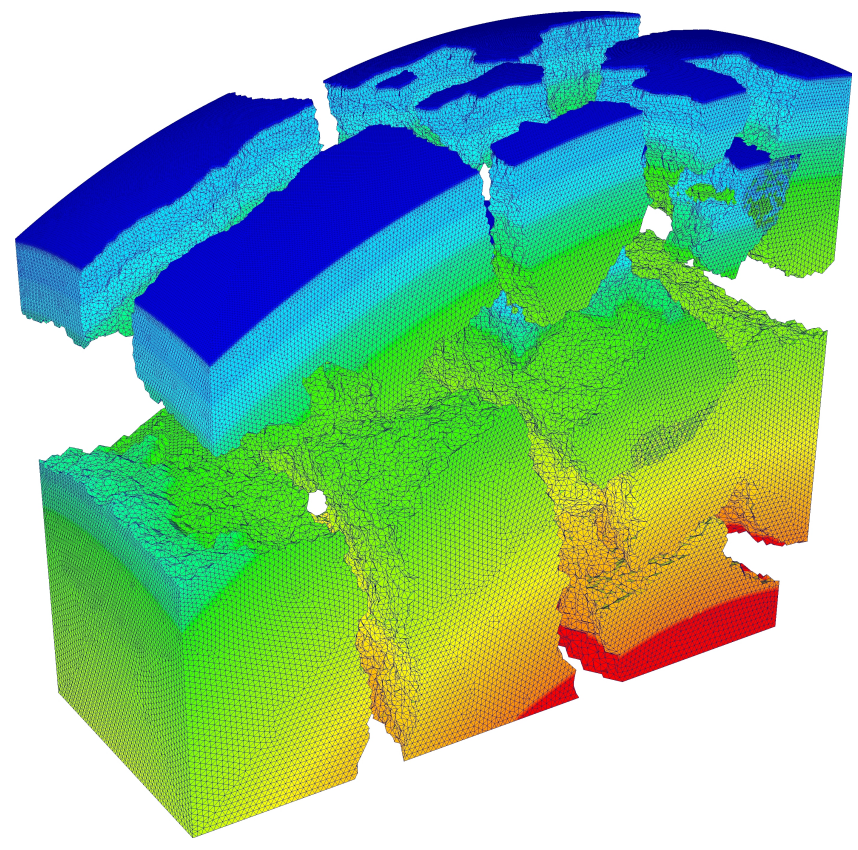

Fig. 4. Computational mesh for benchmark simulations using the 1-D isotropic PREM model (density values are color coded). For visualization purposes the mesh is separated into 15 chunks.

Table 2. CMT solution of the 2009 Norwegian Sea event.

\begin{tabular}{ccccccc}
\hline$M_{r r}$ & $M_{\theta \theta}$ & $M_{\varphi \varphi}$ & $M_{r \theta}$ & $M_{r \varphi}$ & $M_{\theta \varphi}$ & Scaling \\
\hline-9.69 & 3.82 & 5.86 & 0.15 & -3.83 & 8.11 & $10^{17} \mathrm{Nm}$ \\
\hline
\end{tabular}

Castro et al. (2010) demonstrated that an element-wise material sampling using constant parameters is not an intrinsic restriction of the DG approach. In the case of the PREM model, the relatively low material gradient (max. $1.5 \%$ velocity variation in one element) is already accurately sampled if the spatial discretization is adapted to the smallest wavelength. The smallest wavelength has to be resolved by the simulation in consideration of the propagation distance of the signal. That means that the sampling of the material distribution does not require a smaller mesh spacing even when a constant material approximation is used.

At near offset stations, larger envelope misfits occurred, which are thought to be caused by the radiation of the source pulse within an asymmetric tetrahedral element. This is connected to the radiation of SH-energy obtained for an explosive source in the SeisSol simulations, which in theory is not allowed. Käser and Igel (2001) claimed that, for an explosive source excited in an isotropic elastic medium, the application of numerical operators on unstructured meshes can lead to S-wave artifacts at the transverse velocity component $v_{t}$. The convergence tests we carried out in an isotropic elastic medium have shown a decreasing $v_{t}$ signal amplitude with decreasing mesh spacing, i.e., the effect is purely numeri- cal and can be diminished by refining the mesh around the source.

The performance of the numerical implementations was estimated by a theoretical calculation of de la Puente et al. (2008). The analysis is reduced to the number of matrix operations $\varnothing$ of SeisSol and SpecFEM, which led to an estimation of $\emptyset_{\mathrm{DG}} \approx 2.8 \cdot \emptyset_{\mathrm{SE}}$ at a polynomial degree of $N=3$. As mentioned in the thesis, one has to be aware that the performance of a software additionally depends, for example, on the programming, the size of the time step, number of elements, number of field variables and the cost of the time integration. Since the simulations of this study provide a different level of accuracy on different meshes and physical domains, a quantitative comparison is not possible in fair terms.

To quantitatively analyze the efficiency of numerical codes, a conceptual basis has to be defined based on an accurate definition of the problem to be solved and the criteria of comparison. For example, errors due to the order of spatial and temporal discretization in the numerical algorithm can be independently dominating, depending on the modeling parameters such as the applied Earth model or the wave propagation distance. These tradeoffs must be explored separately and will be addressed in a future study to evaluate the ADER-DG and SE code efficiency.

\section{Application of the ADER-DG method to real data: The 2009 L'Aquila earthquake}

In the previous tests, it could be demonstrated that SeisSol is able to accurately simulate elastic wave propagation on layered 1-D Earth models like PREM (viscoelastic effects are neglected). In this section, we qualitatively compare SeisSol synthetics with real data of the magnitude $M_{W}=6.3$ L'Aquila event (central Italy) to examine the applicability of the ADER-DG method on modeling real earthquake scenarios using 3-D Earth models.

The earthquake source area evolved by subduction of the Adria micro-plate, by continental collision of Eurasia and Africa, and by the opening of the Tyrrhenian back-arc basin. Therefore, the region of the central Apennines is tectonically very complex, and thus has one of the highest seismic hazard records in Italy (Chiarabba et al., 2009).

On the 6th of April 2009 (01:32 GMT) the main shock of a seismic sequence struck the Abruzzi region due to normal faulting along a NW-SE striking fault system at a depth of about $12 \mathrm{~km}$. The earthquake devastated the city of L'Aquila and surrounding villages, leading to 308 deaths, about 1500 injured and an economic loss of 15 billion Euro.

\subsection{Model setup}

To consider a realistic representation of the Earth's crust, we introduce lateral variations of the elastic material values using the recent 3-D model EPcrust of Molinari and Morelli 


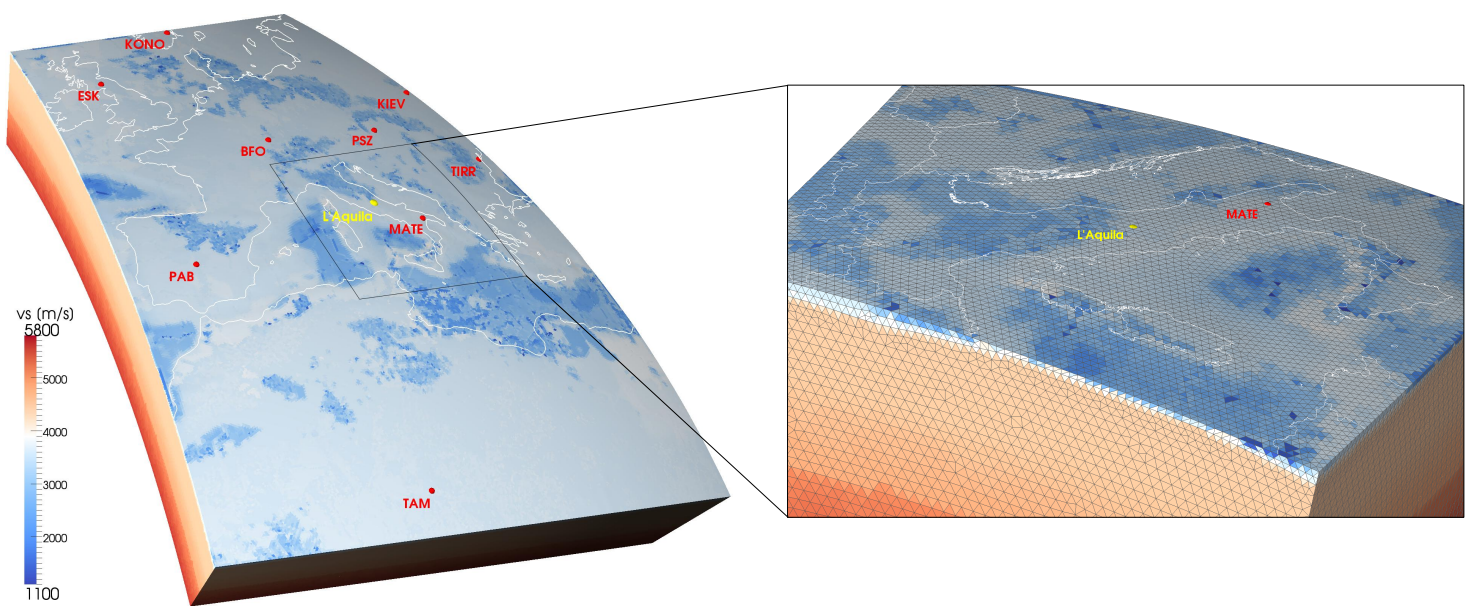

Fig. 5. European model composed of the 3-D EPcrust model of Molinari and Morelli (2011) and the 1-D ak135 model of Kennett et al. (1995) (left). The computational domain is subdivided into a mesh of unstructured tetrahedral elements (right). 3-D Topography of the Earth surface and the crust-mantle boundary is respected by the mesh.

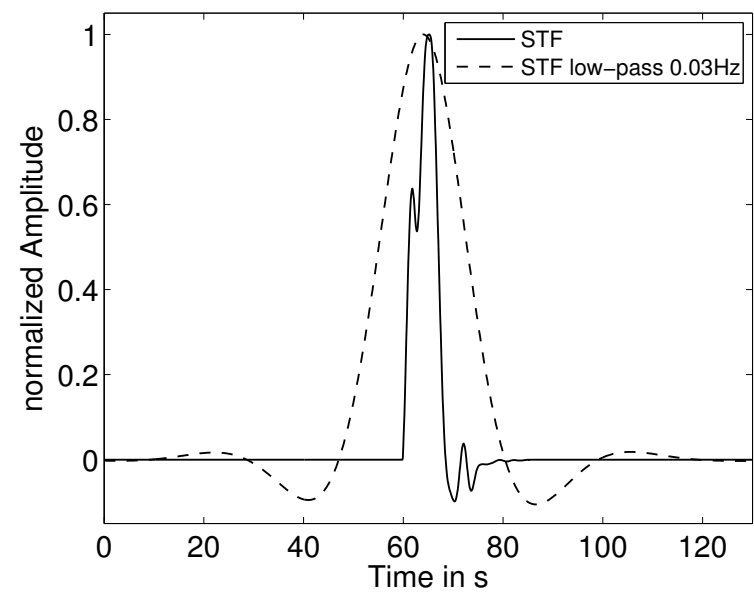

Fig. 6. Inverted source time function of the 2009 L'Aquila event unfiltered (solid) and filtered to periods of $T \geq 33 \mathrm{~s}$ (dashed).

(2011). This model is imposed on top of the 1-D reference Earth model akl35 of Kennett et al. (1995) to form a composite model of Europe for the ADER-DG simulations.

The EPcrust model was deduced from literature information of different global and local models using active-source seismic profiles, receiver function studies and digital maps (e.g., Bassin et al., 2000; Tesauro et al., 2008). It is divided into 3 layers (sediments, upper and lower crust) of variable thickness, which cover the whole European Plate on a geographical grid of $0.5^{\circ} \times 0.5^{\circ}$. Surface topography is incorporated by the ETOPO1 model of Amante and Eakins (2008). The geologic formations are parametrized on curved layer interfaces by the isotropic P-, S-wave velocity and density. The $\mathrm{P}$-wave velocity varies between $1.5<v_{P}<7.2 \mathrm{~km} \mathrm{~s}^{-1}$, while, due to scaling relations of Brocher (2005), the Swave velocity and density range by $0.4<v_{S}<4.1 \mathrm{~km} \mathrm{~s}^{-1}$ and $0.9<\rho<3.0 \mathrm{~g} \mathrm{~cm}^{-3}$, respectively.
The 1-D reference model akl35 (Kennett et al., 1995) was constructed from a set of empirical traveltime curves of relocated earthquake events. It provides good fits to a variety of seismic phases, but does not respect the effect of lateral heterogeneities inside the Earth. In our European model, ak135 supplies the upper mantle $(35-660 \mathrm{~km})$ material values for P-wave velocity $8.0<v_{P}<10.2 \mathrm{~km} \mathrm{~s}^{-1}$, $\mathrm{S}$-wave velocity $4.5<v_{S}<5.6 \mathrm{~km} \mathrm{~s}^{-1}$ and density $3.3<\rho<4.1 \mathrm{~g} \mathrm{~cm}^{-3}$.

\subsection{Geometrical representation and mesh generation}

The physical representation used for the ADER-DG simulation is spherically bounded by $20^{\circ} \mathrm{N}-60^{\circ} \mathrm{N}$ latitude and $10^{\circ} \mathrm{W}-30^{\circ} \mathrm{E}$ longitude, shown in the left sketch of Fig. 5. In this domain, the topography of the Earth surface and the Mohorovičić discontinuity (Moho) are represented by 3-D splines. They form the top and bottom of the crustal layer with a thickness of $6.3-52.1 \mathrm{~km}$, while interior interfaces of the upper and lower crust are not respected. The upper mantle region is composed of three layers bounded by smooth spherical interfaces according to the elastic discontinuities in $a k 135$ at $210,410,660 \mathrm{~km}$ depth.

To generate a mesh inside the volume, an appropriate mesh size has to be defined. The specification depends on geometrical restrictions of the physical domain, the prospective approximation accuracy of the material model, and the spatial sampling of the wavefield. According to an average minimum crustal velocity (PREM model) of $v_{S}=3200 \mathrm{~m} \mathrm{~s}^{-1}$, the number of propagated wavelengths is assumed to be $\# \lambda \approx 20$. Due to Käser et al. (2008), we expect high simulation accuracies over regional propagation distances at a spatial sampling of 2 tetrahedral elements per smallest wavelength if an $\varnothing=5$ scheme is applied.

As already mentioned, in SeisSol, the material values of tetrahedral elements are averaged over all vertex values. Therefore, using an average element edge length of $18 \mathrm{~km}$ for 


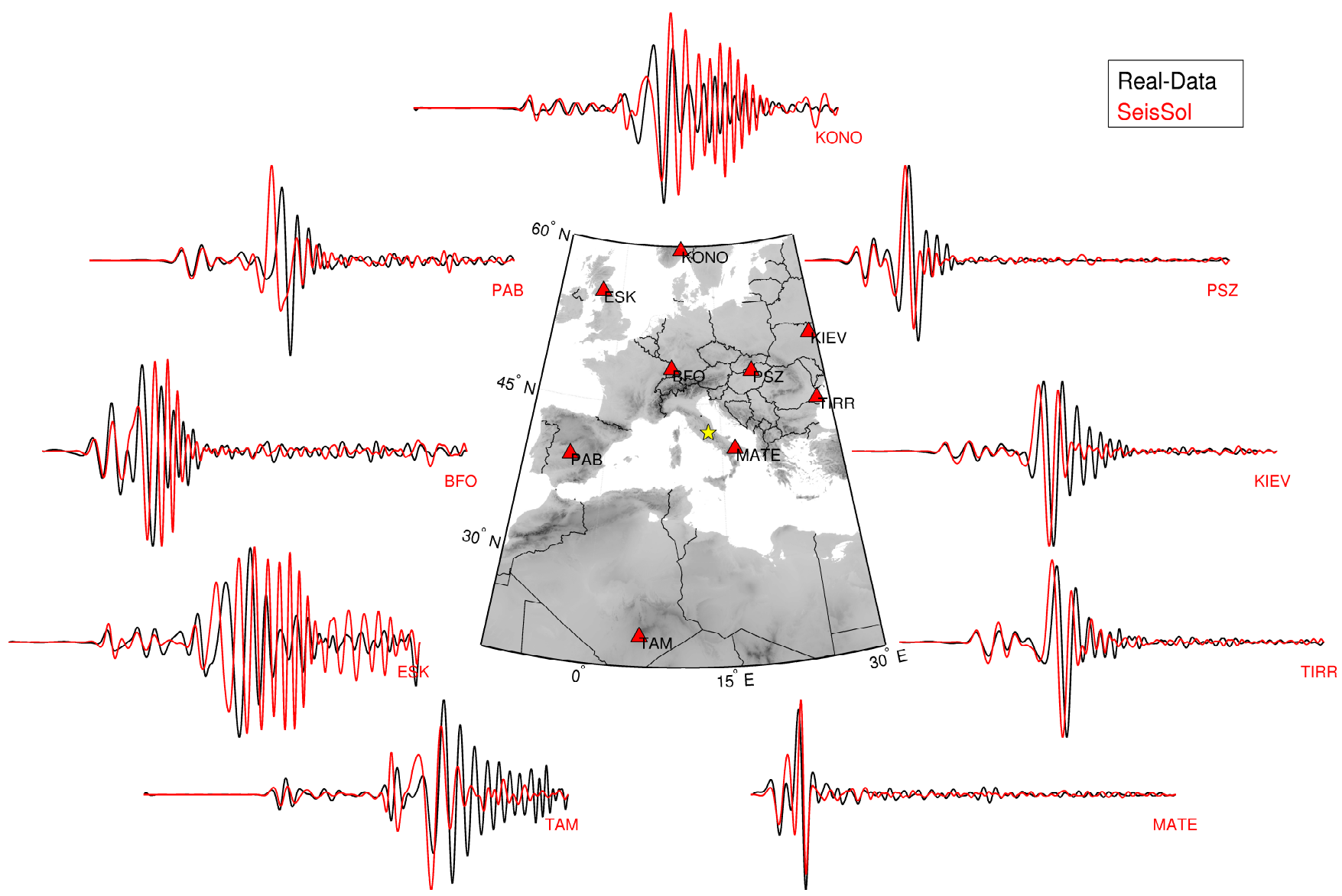

Fig. 7. Synthetic seismograms of the ADER-DG method obtained on the European model shown in Fig. 5 compared to real data of the 2009 L'Aquila earthquake (yellow star) at 9 different stations (red triangles). The vertical velocity component is shown for seismograms of $800 \mathrm{~s}$ length, filtered to periods of $T \geq 33 \mathrm{~s}$.

the crustal mesh generation, the interpolation of material values smooths the underlying crustal model, which leads to a lowest surface wave velocity of $1.1 \mathrm{~km} \mathrm{~s}^{-1}$. Assuming a seismic source signal at a dominant period of $33 \mathrm{~s}$, the smallest wavelength of $36 \mathrm{~km}$ can be sampled correctly. The resulting mesh incorporates high-quality tetrahedral elements, as illustrated in the enlarged sketches of Fig. 5.

The significant change in the velocity model cannot be avoided, unless the sedimentary layer is respected explicitly in the mesh, or an inhomogeneous material distribution inside elements is applied in cases of implicit meshing. However, this would lead to such a high number of additional elements that the simulation would not be feasible anymore on available HPC resources.

In the mantle, the mesh size is adapted according to the 1D velocity distribution of $a k 135$ to ensure a spatial sampling of 2 elements per shortest wavelength. For the whole mesh, this results in a total number of 3.7 million elements leading to 1164 million degrees of freedom for an $\varnothing 5$ ADERDG simulation. On an IBM system using 50 Intel Xeon Westmere-EX processors (10 cores, $2.4 \mathrm{GHz}$ ), a runtime of $24 \mathrm{~h}$ was necessary to produce output seismograms of $800 \mathrm{~s}$.
Table 3. CMT solution of the 2009 L'Aquila event.

\begin{tabular}{ccccccc}
\hline$M_{r r}$ & $M_{\theta \theta}$ & $M_{\varphi \varphi}$ & $M_{r \theta}$ & $M_{r \varphi}$ & $M_{\theta \varphi}$ & Scaling \\
\hline-3.30 & 1.43 & 1.87 & -1.43 & -0.27 & -1.77 & $10^{18} \mathrm{Nm}$ \\
\hline
\end{tabular}

\subsection{Data processing}

For the L'Aquila earthquake, broadband data of a high signalto-noise ratio are provided by several seismological networks such as IRIS, GEOFON or GEOSCOPE. From these networks, we chose 9 stations (BFO, ESK, KIEV, KONO, MATE, PAB, PSZ, TAM, TIRR) at an epicentral distance of $330-2300 \mathrm{~km}$ azimuthal located around the source; see Figs. 7 and 8 . The event-based raw data were extracted from the data centers and corrected for the instrument response.

Synthetic data is generated using SeisSol applied to the composite European model described above. For the simulation, a source mechanism specified by the centroid moment tensor solution of the event is used; see Table 3. The data processing further involves the convolution by a source time 


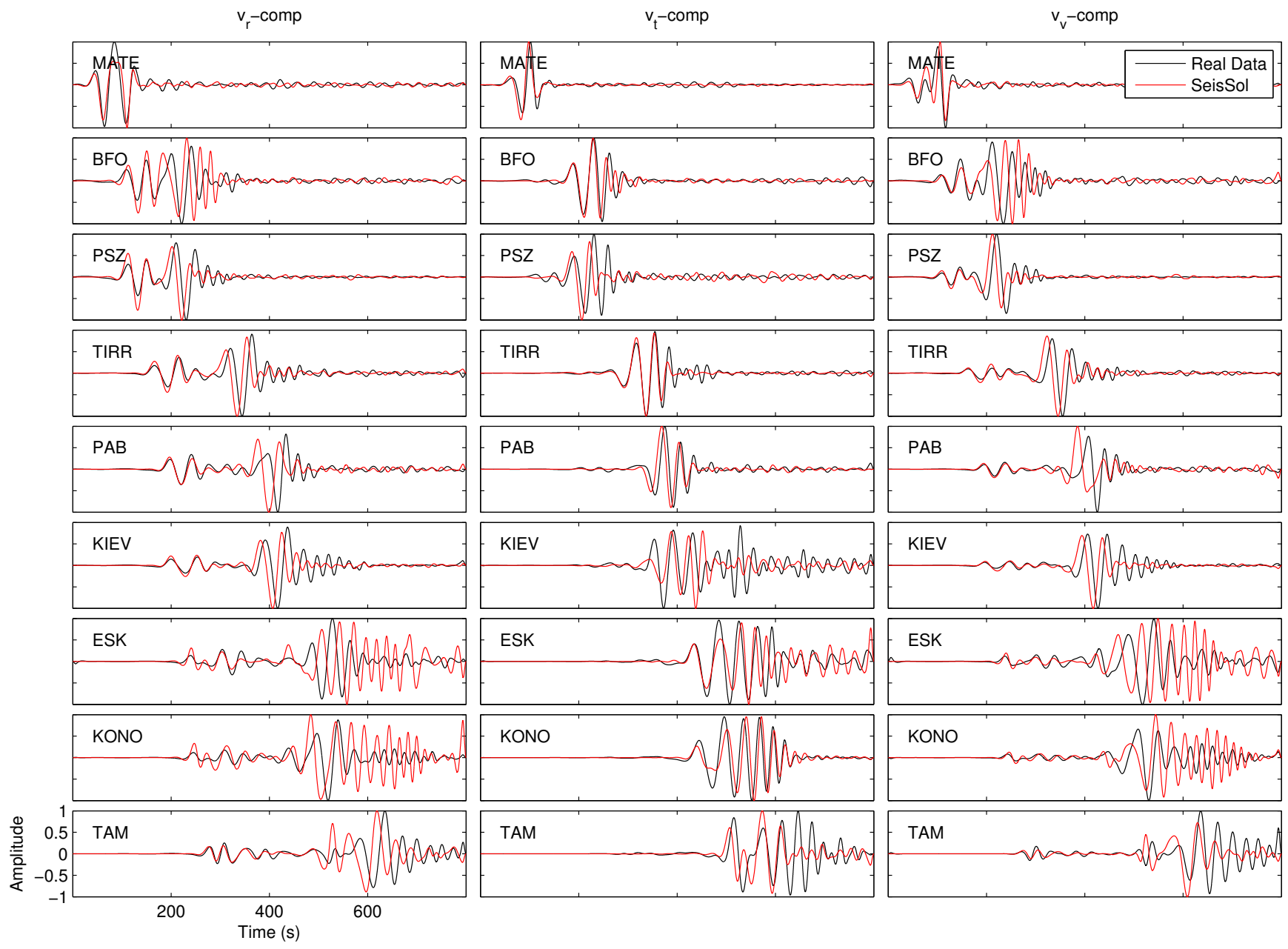

Fig. 8. Synthetic seismograms of the ADER-DG method obtained on the European model shown in Fig. 5 compared to real data of the 2009 L'Aquila earthquake at 9 different stations. The radial, transverse and vertical velocity components are shown for seismograms of $800 \mathrm{~s}$ length, filtered to periods of $T \geq 33 \mathrm{~s}$.

function, as shown in Fig. 6. To obtain the STF, an inversion scheme using a neighbourhood algorithm is applied as described in Stähler et al. (2012). Subsequently, the real and synthetic data are filtered for long period signals at a cut-off period of $33 \mathrm{~s}$.

\subsection{Results and discussion}

For real and synthetic data, the amplitude normalized traces of the vertical velocity component are shown in Fig. 7. Here, we want to emphasize that especially P-wave phases remarkably fit the measured phases at all observed stations. Surface wave arrivals usually show a larger misfit in phase and amplitude compared to real data. However, the overall signal duration matches well using the inverted source time function. The misfit between data and synthetics can be attributed mainly to the approximation of the material values inside the Earth by the applied velocity models. As already mentioned, also the fact that not every small feature can be modeled exactly with the given resolution (e.g., shallow low velocity zones) can have an influence on the simulation results. Since the surface waves tend to arrive earlier in the simulations, which suggests an overestimation of the velocities, an influence of the model discretization cannot be excepted.

Concluding, we also want to mention that an interference of the synthetics by artificial boundary reflections cannot be excepted; clear signal onsets could not be identified.

\section{Conclusions}

We presented a study to verify the application of the ADERDG method for regional scale earthquake simulations. In particular, the flexibility of unstructured tetrahedral meshing allows the creation of high-quality computational grids to study wave propagation in 3-D media.

In two benchmark tests obtained using a 1-D isotropic PREM model, we quantitatively evaluated wave propagation 
over intermediate distances $\left(<15^{\circ}\right)$ using an explosive and a shear dislocation source. In comparison to the spectralelement method, we could show that the ADER-DG method provides accurate synthetic results of low phase and envelope misfits. Due to the use of unstructured tetrahedral meshes, numerical artefacts were obtained, which decrease in amplitude for successive mesh refinements.

In the second part, the simulation of the L'Aquila earthquake confirms the applicability of the method using a regional scale 3-D composite model of Europe. The results expose an excellent fit of first arrival P-wave signals with real data measurements at a shortest period of $33 \mathrm{~s}$. Surface waves, most sensitive to crustal structures, exhibit phase and amplitude misfits, indicating the deficiencies in the representation of the material values inside the Earth using the EPcrust and ak135 model.

As described in Sect. 2.2, the design of unstructured tetrahedral meshes is fast and flexible. In addition, features like $p$-adaptivity and local time stepping can focus the computational effort using the ADER-DG method. This allows for accurate simulations in regions such as the Alps where strong lateral heterogeneities in the material distribution as well as geometrical complexities due to surface and interface topography strongly perturb 3-D wave propagation. In a future study, we want to assess the quality of new Earth models and explore in detail the benefits of the local spatial and temporal adaptivity provided by the ADER-DG algorithm.

Acknowledgements. This work was funded by the Bavarian Government through the KONWIHR Geo-PF project and the graduate school THESIS as well as the Volkswagen Foundation, which supported our work through the ASCETE project (AZ 85433). The authors also want to thank the Leibniz-Rechenzentrum (LRZ) and our system administrator Jens Oeser for access and support in using the HLRB-II system at the LRZ and the local institute cluster TETHYS (Oeser et al., 2006). We thank the QUEST international training network funded by the European Commission, which provided an excellent research environment and also financial support especially for young scientists. Furthermore, the authors want thank the reviewers, especially Vincent Etienne, for their thorough comments which helped to improve the manuscript.

Edited by: V. Sallares

\section{References}

Alterman, Z., Jarosch, H., and Pekeris, C. L.: Oscillations of the Earth, Proc. R. Soc. Lond. A, 252, 80-95, http://rspa. royalsocietypublishing.org/content/252/1268/80.sh\%ort, 1959.

Amante, C. and Eakins, B. W.: ETOPO1 1 Arc-Minute Global Relief Model: Procedures, Data Sources and Analysis, National Geophysical Data Center, NESDIS, NOAA, U.S. Department of Commerce, Boulder, CO, 2008.

Bao, H., Bielak, J., Ghattas, O., Kallivokas, L. F., O'Hallaron, D. R., Shewchuk, J. R., and Xu, J.: Large-scale simulation of elastic wave propagation in heterogeneous media on parallel computers, Comp. Methods Appl. Mech. Eng., 152, 85-102, doi:10.1016/S0045-7825(97)00183-7, 1998.

Bassin, C., Laske, G., and Masters, G.: The current limits of resolution for surface wave tomography in North America, EOS Trans. AGU, 81, F897, 2000.

Bayliss, A., Jordan, K. E., Lemesurier, B. J., and Turkel, E.: A fourth-order accurate finite-difference scheme for the computation of elastic waves, Bull. Seism. Soc. Am., 76, 1115-1132, http://www.bssaonline.org/cgi/content/abstract/76/4/1115, 1986.

Blacker, T. D., Bohnhoff, W. J., and Edwards, T. L.: CUBIT mesh generation environment. Volume 1: Users manual, Tech. rep., Sandia National Labs., Albuquerque, NM (United States), doi:10.2172/10176386, 1994.

Bohlen, T. and Saenger, E. H.: Accuracy of heterogeneous staggered-grid finite-difference modeling of Rayleigh waves, Geophysics, 71, 109-115, 2006.

Brocher, T. M.: Empirical Relations between Elastic Wavespeeds and Density in the Earth's Crust, Bull. Seism. Soc. Am., 95 , 2081-2092, doi:10.1785/0120050077, http://www.bssaonline. org/cgi/content/abstract/95/6/2081, 2005.

Capdeville, Y., Vilotte, J.-P., and Montagner, J.-P.: Coupling the spectral element method with a modal solution for elastic wave propagation in global earth models, Geophys. J. Int., 152, 34-67, doi:10.1046/j.1365-246X.2003.01808.x, 2003.

Casarotti, E., Magnoni, F., Le Goff, N., Martin, R., Komatitsch, D., Plesch, A., Nissen-Meyer, T., Luo, Y., and Tromp, J.: Mesh Generation for Short-Period Seismic Wave Propagation Based Upon the Spectral- Element Method: Southern California., AGU Fall Meeting Abstracts, 89, S61B-1119, http://adsabs.harvard. edu/abs/2008AGUFM.S23A1875C, 2008a.

Casarotti, E., Stupazzini, M., Lee, S. J., Komatitsch, D., Piersanti, A., and Tromp, J.: CUBIT and seismic wave propagation based upon the Spectral-Element Method: An advanced unstructured mesher for complex 3D geological media, Vol. 5B.4, Chap. CUBIT and, 579-597, Springer, Berlin, 2008b.

Castro, C. E., Käser, M., Brietzke, G. B., C. E. Castro, and G. B. Brietzke: Seismic waves in heterogeneous material: subcell resolution of the discontinuous Galerkin method, Geophys. J. Int., 182, 250-264, doi:10.1111/j.1365-246X.2010.04605.x, 2010.

Chaljub, E.: Modélisation numériques de la propagation d'ondes sismiques à l'échelle du globe, Phd thesis, Université Paris 7, 2000.

Chiarabba, C., Amato, a., Anselmi, M., Baccheschi, P., Bianchi, I., Cattaneo, M., Cecere, G., Chiaraluce, L., Ciaccio, M. G., De Gori, P., De Luca, G., Di Bona, M., Di Stefano, R., Faenza, L., Govoni, a., Improta, L., Lucente, F. P., Marchetti, a., Margheriti, L., Mele, F., Michelini, a., Monachesi, G., Moretti, M., Pastori, M., Piana Agostinetti, N., Piccinini, D., Roselli, P., Seccia, D., and Valoroso, L.: The 2009 L'Aquila (central Italy) M W 6.3 earthquake: Main shock and aftershocks, Geophys. Res. Lett., 36, 1-6, doi:10.1029/2009GL039627, 2009.

Cupillard, P., Delavaud, E., Burgos, G., Festa, G., Vilotte, J.-P., Capdeville, Y., and Montagner, J.-P.: RegSEM: a versatile code based on the spectral element method to compute seismic wave propagation at the regional scale, Geophys. J. Int., 188, 12031220, doi:10.1111/j.1365-246X.2011.05311.x, 2012.

de la Puente, J., Käser, M., Dumbser, M., and Igel, H.: An arbitrary high-order discontinuous Galerkin method for elastic waves 
on unstructured meshes - IV. Anisotropy, Geophys. J. Int., 169, 1210-1228, doi:10.1111/j.1365-246X.2007.03381.x, 2007.

de la Puente, J., Castro, C. E., and Hermann, V.: Seismic wave field modelling using high performance computing, in: SEG Technical Program Expanded Abstracts, 2884-2888, http://link.aip.org/ link/?SEGEAB/27/2884/1, 2008.

Dubiner, M.: Spectral methods on triangles and other domains, J. Sci. Comput., 6, 345-390, http://www.springerlink.com/index/ T5115604621W526L.pdf, 1991.

Dumbser, M. and Käser, M.: An arbitrary high-order discontinuous Galerkin method for elastic waves on unstructured meshes - II, The three-dimensional isotropic case, Geophys. J. Int., 167, 319 336, doi:10.1111/j.1365-246X.2006.03120.x, 2006.

Dumbser, M., Käser, M., and Toro, E. F.: An arbitrary highorder Discontinuous Galerkin method for elastic waves on unstructured meshes - V. Local time stepping and p adaptivity, Geophys. J. Int., 171, 695-717, doi:10.1111/j.1365246X.2007.03427.x, 2007.

Dziewonski, A. M. and Anderson, D. L.: Preliminary reference Earth model, Phys. Earth Planet. Int., 25, 297-356, doi:10.1016/0031-9201(81)90046-7, 1981.

Ely, G. P., Day, S. M., and Minster, J.-B.: A support-operator method for viscoelastic wave modelling in 3-D heterogeneous media, Geophysical Journal International, 172, 331-344, doi:10.1111/j.1365-246X.2007.03633.x, 2008.

Etienne, V., Chaljub, E., Virieux, J., and Glinsky, N.: An hpadaptive discontinuous Galerkin finite-element method for 3D elastic wave modelling, Geophys. J. Int., 183, 941-962, doi:10.1111/j.1365-246X.2010.04764.x, 2010.

Fichtner, A.: Full seismic waveform inversion for structural and source parameters, Phd thesis, LMU Munich, http://edoc.ub. uni-muenchen.de/11494/1/fichtner_andreas.pdf, 2009.

Fichtner, A. and Igel, H.: Efficient numerical surface wave propagation through the optimization of discrete crustal modelsa technique based on non-linear dispersion curve matching (DCM), Geophys. J. Int., 173, 519-533, doi:10.1111/j.1365246X.2008.03746.x, 2008.

Fichtner, A., Igel, H., Bunge, H.-p., and Kennett, B. L. N.: Simulation and Inversion of Seismic Wave Propagation on Continental Scales Based on a Spectral-Element Method, Journal of Numerical Analysis, Industrial and Applied Mathematics, 4, 11-22, 2009.

George, P., Hecht, F., and Saltel, E.: Fully automatic mesh generator for 3D domains of any shape, IMPACT of Computing in Science and Engineering, 2, 187-218, doi:10.1016/0899-8248(90)90012Y, 1990.

Gustafsson, B. and Wahlund, P.: Time Compact Difference Methods for Wave Propagation in Discontinuous Media, SIAM J. Sci. Comput., 26, 272, doi:10.1137/S1064827503425900, 2004.

Igel, H.: Wave propagation in three-dimensional spherical sections by the Chebyshev spectral method., Geophys. J. Int., 136, 559566, 1999.

Igel, H., Nissen-Meyer, T., and Jahnke, G.: Wave propagation in 3D spherical sections: effects of subduction zones, Phys. Earth Planet. Int., 132, 219-234, doi:10.1016/S0031-9201(02)000535, 2002.

Karypis, G. and Kumar, V.: Multilevel k-way partitioning scheme for irregular graphs, J. Parallel Distrib. Comput., 48, 96-129, 1998.
Käser, M. and Dumbser, M.: An Arbitrary High Order Discontinuous Galerkin Method for Elastic Waves on Unstructured Meshes I: The Two-Dimensional Isotropic Case with External Source Terms, Geophys. J. Int., 166, 855-877, 2006.

Käser, M. and Igel, H.: Numerical simulation of 2D wave propagation on unstructured grids using explicit differential operators, Geophys. Prospect., 49, 607-619, 2001.

Käser, M., Dumbser, M., de la Puente, J., and Igel, H.: An arbitrary high-order Discontinuous Galerkin method for elastic waves on unstructured meshes - III. Viscoelastic attenuation, Geophys. J. Int., 168, 224-242, doi:10.1111/j.1365-246X.2006.03193.x, 2007.

Käser, M., Hermann, V., and de la Puente, J.: Quantitative Accuracy Analysis of the Discontinuous Galerkin Method for Seismic Wave Propagation, Geophys. J. Int., 173, 990-999, doi:10.1111/j.1365-246X.2008.03781.x, 2008.

Käser, M., Pelties, C., Castro, C. E., Djikpesse, H., and Prange, M.: Wavefield modeling in exploration seismology using the discontinuous Galerkin finite-element method on HPC infrastructure, The Leading Edge, 29, 76, http://tle.geoscienceworld.org/ cgi/content/abstract/29/1/76, 2010.

Kennett, B. L. N., Engdahl, E. R., and Buland, R.: Constraints on seismic velocities in the Earth from traveltimes, Geophys. J. Int., 122, 108-124, doi:10.1111/j.1365-246X.1995.tb03540.x, 1995.

Komatitsch, D.: Méthodes spectrales et éléments spectraux pour l'équation de l'élastodynamique $2 \mathrm{D}$ et $3 \mathrm{D}$ en milieu hétérogène (Spectral and spectral-element methods for the 2D and 3D elastodynamics equations in heterogeneous media), Ph.D. thesis, Institut de Physique du Globe, Paris, France, 1997.

Komatitsch, D. and Tromp, J.: Spectral-element simulations of global seismic wave propagation-I. Validation, Geophys. J. Int., 149, 390-412, doi:10.1046/j.1365-246X.2002.01653.x, 2002.

Komatitsch, D., Liu, Q., Tromp, J., Süss, P., Stidham, C., and Shaw, J. H.: Simulations of Ground Motion in the Los Angeles Basin Based upon the Spectral-Element Method, Bull. Seism. Soc. Am., 94, 187-206, doi:10.1785/0120030077, 2004.

Kozdon, J. E., Dunham, E. M., and Nordström, J.: Simulation of Dynamic Earthquake Ruptures in Complex Geometries Using High-Order Finite Difference Methods, J. Sci. Comput., http: //www.springerlink.com/content/e1k0515422872700/, 2012.

Kristek, J. and Moczo, P.: Seismic-Wave Propagation in Viscoelastic Media with Material Discontinuities: A 3D Fourth-Order Staggered-Grid Finite-Difference Modeling, Bull. Seism. Soc. Am., 93, 2273-2280, 2003.

Kristek, J., Moczo, P., and Galis, M.: Stable discontinuous staggered grid in the finite-difference modelling of seismic motion, Geophys. J. Int., 183, 1401-1407, 2010.

Kristekova, M., Kristek, J., Moczo, P., and Day, S. M.: Misfit Criteria for Quantitative Comparison of Seismograms, Bull. Seism. Soc. Am., 96, 1836-1850, doi:10.1785/0120060012, 2006.

Lee, S.-J., Chen, H.-W., Liu, Q., Komatitsch, D., Huang, B.-S., and Tromp, J.: Three-Dimensional Simulations of Seismic-Wave Propagation in the Taipei Basin with Realistic Topography Based upon the Spectral-Element Method, Bull. Seism. Soc. Am., 98, 253-264, doi:10.1785/0120070033, 2008.

Molinari, I. and Morelli, A.: EPcrust: a reference crustal model for the European Plate, Geophys. J. Int., 185, 352-364, doi:10.1111/j.1365-246X.2011.04940.x, 2011. 
Nissen-Meyer, T., Fournier, A., and Dahlen, F. A.: A 2-D spectralelement method for computing spherical-earth seismograms-II. Waves in solid-fluid media, Geophys. J. Int., 174, 873-888, 2008.

Oeser, J., Bunge, H.-P., and Mohr, M.: Cluster Design in the Earth Sciences: TETHYS, in: High Performance Computing and Communications - Second International Conference, HPCC 2006, Munich, Germany, edited by Gerndt, M. and Kranzlmüller, D., Vol. 4208 of Lecture Notes in Computer Science, 31-40, Springer, 2006.

Pelties, C., Käser, M., Hermann, V., and Castro, C. E.: Regular versus irregular meshing for complicated models and their effect on synthetic seismograms, Geophys. J. Int., 183, 1031-1051, doi:10.1111/j.1365-246X.2010.04777.x, 2010.

Peter, D., Komatitsch, D., Luo, Y., Martin, R., Le Goff, N., Casarotti, E., Le Loher, P., Magnoni, F., Liu, Q., Blitz, C., Nissen-Meyer, T., Basini, P., and Tromp, J.: Forward and adjoint simulations of seismic wave propagation on fully unstructured hexahedral meshes, Geophys. J. Int., 186, 721-739, doi:10.1111/j.1365-246X.2011.05044.x, 2011.

Pitarka, A.: 3D Elastic finite-difference modeling of seismic motion using staggered grids with nonuniform spacing, Bull. Seis. Soc. Am., 89, 54-68, 1999.

Priolo, E., Carcione, J. M., and Seriani, G.: Numerical simulation of interface waves by high-order spectral modeling techniques, The Journal of the Acoustical Society of America, 95, 681, doi:10.1121/1.408428, 1994.

Ronchi, C.: The Cubed Sphere: A New Method for the Solution of Partial Differential Equations in Spherical Geometry, J. Comp. Phys., 124, 93-114, doi:10.1006/jcph.1996.0047, 1996.
Seriani, G.: 3-D large-scale wave propagation modeling by spectral element method on Cray T3E multiprocessor, Comp. Methods Appl. Mech. Eng., 164, 235-247, doi:10.1016/S00457825(98)00057-7, 1998.

Stähler, S. C., Sigloch, K., and Nissen-Meyer, T.: Triplicated Pwave measurements for waveform tomography of the mantle transition zone, Solid Earth, 3, 339-354, doi:10.5194/se-3-3392012, 2012.

Stupazzini, M., Paolucci, R., and Igel, H.: Near-Fault Earthquake Ground-Motion Simulation in the Grenoble Valley by a HighPerformance Spectral Element Code, Bull. Seis. Soc. Am., 99, 286-301, doi:10.1785/0120080274, http://www.bssaonline.org/ cgi/content/abstract/99/1/286, 2009.

Tesauro, M., Kaban, M. K., and Cloetingh, S. A. P. L.: EuCRUST07: A new reference model for the European crust, Geophys. Res. Lett., 35, L05313, doi:10.1029/2007GL032244, 2008.

Toro, E. F.: Riemann Solvers and Numerical Methods for Fluid Dynamics, Springer, Berlin, 2 Edn., http://www.springer.com/ materials/mechanics/book/978-3-540-25202-3, 1999.

Virieux, J.: SH-wave propagation in heterogeneous media: Velocity-stress finite-difference method, Geophysics, 49, 1933, doi:10.1190/1.1441605, 1984.

Woodhouse, J. H.: The joint inversion of seismic waveforms for lateral variations in Earth structure and earthquake source parameters, in: Proceedings of the Enrico Fermi International School of Physics, edited by: Kanamori, H. and Boschi, E., 366-397, North-Holland Netherlands, Amsterdam, 1983. 